\title{
Evaluation of Sarcopenia and Investigation of Prognostic Value Of Sarcopenia Using Psoas Muscle Area on Computed Tomography in Patients with Liver Cirrhosis
}

\author{
Merve Erkan ${ }^{1^{*}}$ (D) Ali Ahmetoglu 2 (D), Aysegul Cansu 2 (D), Murat Erkut ${ }^{3}$ \\ ${ }^{1}$ Health Sciences University Bursa Yuksek Ihtisas Training and Research Hospital, Department of Radiology, Bursa, Turkey \\ ${ }^{2}$ Karadeniz Technical University, Faculty of Medicine, Department of Radiology, Trabzon, Turkey \\ ${ }^{3}$ Karadeniz Technical University, Faculty of Medicine, Department of Gastroenterology, Trabzon, Turkey \\ * Corresponding author: Merve Erkan E-mail: merveaksoy86@hotmail.com ORCID: 0000-0002-9261-7125 \\ Received: 6 October 2020 Accepted: 3 July 2021
}

\begin{abstract}
Objective: To investigate the frequency of sarcopenia in cirrhotic patients, the correlation of sarcopenia with conventional prognostic scores and its relationship with mortality by measuring the psoas muscle area at the level of mid-3rd lumbar (L3) vertebra on computed tomography (CT) scan.

Materials and Methods: We included 229 cirrhotic patients who had undergone abdominal CT scan for hepatocellular carcinoma screening. A control group was formed in 204 healthy individuals who had undergone abdominal computed tomography examination due to trauma. 1- and 3-year follow-up results were recorded. Cross-sectional areas of the right and left psoas muscles were calculated in axial sections passing through the level of the mid-L 3 in CT images. The cases with two standard deviations under the mean of the total psoas muscle area in control group were evaluated as sarcopenic.
\end{abstract}

Results: Sarcopenia was detected in $39.7 \%$ of 229 cirrhotic patients. While a statistically significant difference was detected in the Child-Pugh prognostic score between cirrhotic patients with and without sarcopenia, there was a modest difference in the MELD score. The percentage of cirrhotic patients with sarcopenia who passed during 1- and 3-year follow-ups (28.6\% and $40.7 \%$, respectively) were significantly higher than non-sarcopenic patients ( $9.4 \%$ and $24.6 \%$, respectively).

Conclusion: The findings obtained in this study suggest that psoas muscle atrophy shown by CT scan image at the level of the mid-L3 vertebra has predictive value for mortality in cirrhotic patients.

Keywords: Cirrhosis, sarcopenia, mortality, computed tomography, psoas muscle area

\section{INTRODUCTION}

Sarcopenia is a syndrome characterised by loss of skeletal muscle mass and strength associated with risks of functional capacity, declining life quality and death [1]. Sarcopenia is associated with aging, nonetheless it has been shown to have a critical relationship with various clinical conditions, like malnutrition, chronic diseases and malignancy [1]. 
Overall, muscle mass in sarcopenia is defined as being below two standard deviations of an average healthy young adult [2].

Liver cirrhosis is one of major causes of death in many regions of the world and in our country. In cirrhotic patients, life threatening complications, for instance variceal bleeding due to portal hypertension, ascites and spontaneous bacterial peritonitis, hepatic encephalopathy and liver failure may occur if they are not treated promptly [3]. Indeed, skeletal muscle depletion is a common feature of malnutrition in cirrhotic patients [4].

Despite high prevalence and important prognostic role of sarcopenia in cirrhosis, it often remains unnoticed. This is because nutritional assessment in these patients can be difficult due to fluid retention and/or patients' overweight $[5,6]$. The evaluation of skeletal muscle wasting by computed tomography (CT) scan has several advantages over traditional diagnostic methods for sarcopenia. Apparently, it is objective and effortless and not influenced by hepatic synthetic dysfunction or salt and water retention [7-9]. CT is commonly used to screen hepatocellular carcinoma (HCC) in cirrhotic patients, and it provides higher reproducibility and objectiveness.

In this study, our objectives were to evaluate the frequency of sarcopenia in cirrhotic patients, the correlation of sarcopenia with conventional prognostic scores and its relationship with mortality by measuring the total psoas muscle area (PMA) on CT scan at the level of mid-3rd lumbar (L3) vertebra.

\section{MATERIALS AND METHODS}

We evaluated 282 adult patients who had diagnosis of liver cirrhosis by liver biopsy and / or clinical, laboratory and radiological examination between September 2012 and April 2014 and underwent abdominal CT scan for HCC

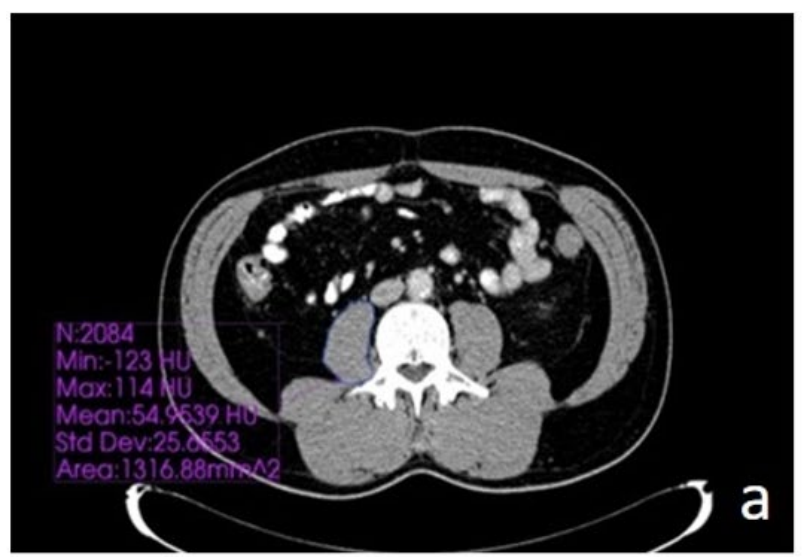

screening at the Black Sea Technical University, Faculty of Medicine, Farabi Hospital, Radiology Department. Fiftythree patients with a concomitant primary malignancy were excluded from the study. A control group comprising 204 age- and gender- matched healthy individuals was formed; these patients were those who did not have a known additional disease and had visited the emergency department for trauma. Local ethics committee approval was obtained for the study. Informed written consent was obtained from the patients.

Age, gender, body weight, height, etiology of cirrhosis, presence of ascites and / or encephalopathy and presence of HCC and laboratory values were screened. In patients diagnosed as cirrhosis, Child-Pugh and MELD scoring systems were used to assess the severity of the liver disease. Clinical and laboratory data were obtained from records within 1 week of CT examination. The 1- and 3-year followup results of the patients were obtained from file data. Patients were divided into two groups as alive and died during 1 and 3 years follow up.

Computerized tomography examinations were performed with multi-section computed tomography (MSCT) devices with 16 detectors (Somatom Sensation, Siemens, Erlangen, Germany) and 160 detectors (Aquillion Prime, Toshiba Medical Systems, Tokyo, Japan). The images were analyzed through picture archiving and communication systems (PACS). In the CT images obtained in our study, the borders of the right and left psoas muscles were drawn in the axial sections passing through the middle part of the $L 3$ vertebra. The total area of the resulting closed areas was automatically calculated to get cross-sectional area of the psoas muscles. Our morphometric measurements were calculated similarly in the control group of 204 trauma patients. Patients under two standard deviations of the mean of the total PMA in the control group were determined as sarcopenic (Figures 1 and 2).

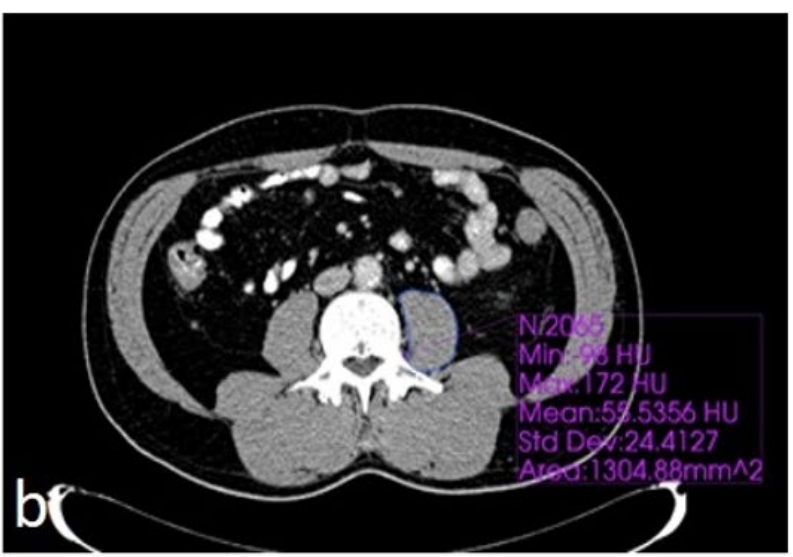

Figure 1. Right (a) and left (b) psoas muscle areas at L3 vertebra level in a 50-year-old non-sarcopenic male patient with cirrhosis. Total PMA $2622 \mathrm{~mm}^{2}$. 

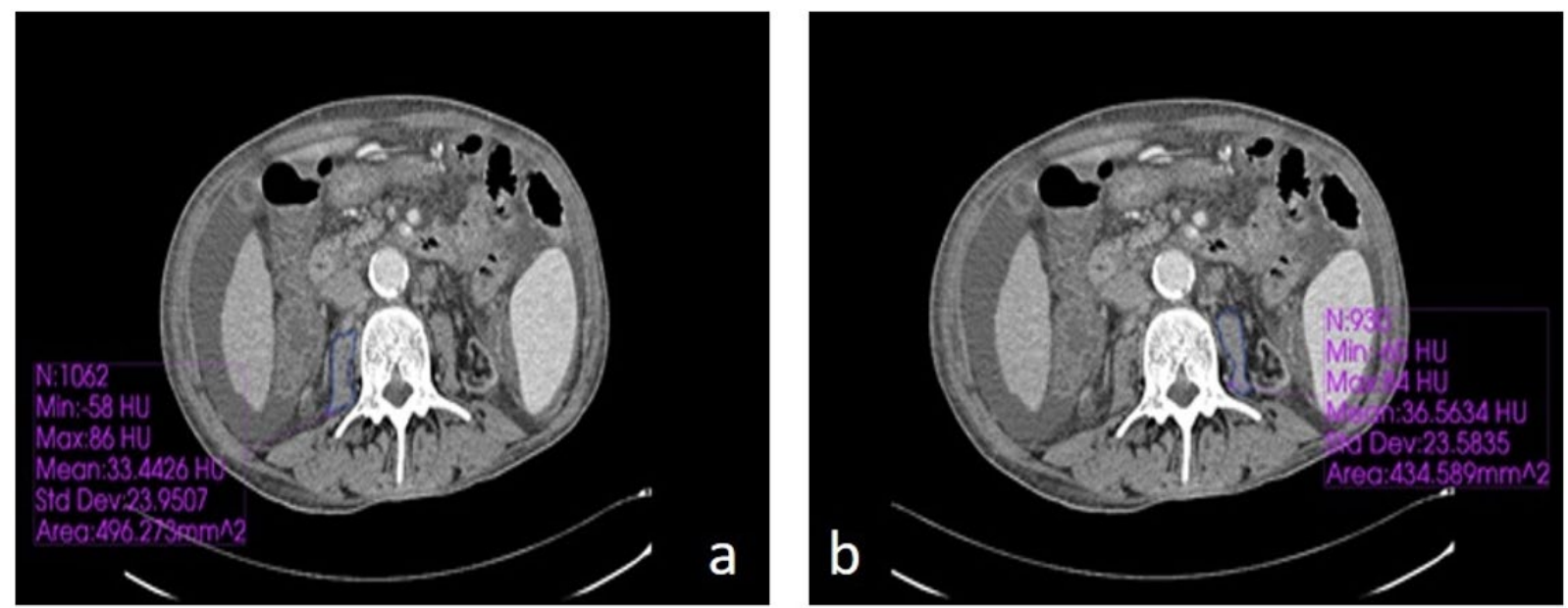

Figure 2. Right (a) and left (b) psoas muscle areas at L3 vertebra level in a 52-year-old sarcopenic male patient with cirrhosis. Total PMA $921 \mathrm{~mm}^{2}$

Table 1. Sarcopenia Cut-Off Values Determined According to the Control Group and the Frequency of Sarcopenia in the Patient Group

\begin{tabular}{|c|c|c|c|c|}
\hline & $\begin{array}{c}\overline{\boldsymbol{X}} \\
\mathbf{( m m )}\end{array}$ & $\begin{array}{c}-\mathbf{2 S} \\
\mathbf{( m m )}\end{array}$ & $\begin{array}{c}\text { Cut-off } \\
(\mathbf{m m})\end{array}$ & \\
\hline Gender & & & & $\begin{array}{c}\text { Sarcopenic Patient } \\
\mathbf{n}(\%)\end{array}$ \\
\hline Male & 2412.9 & -976.8 & 1436.1 & $51(36,4)$ \\
\hline Female & 1406.1 & -487.6 & 918.5 & $40(44.9)$ \\
\hline TOTAL & & & & $91(39.7)$ \\
\hline
\end{tabular}

\section{Statistical Analysis}

The research data were analyzed with SPSS 22 statistical software (SPSS Inc, Chicago, IL). Data were expressed as mean \pm standard deviation, frequency distribution and percentage. Categorical variables were assessed using ChiSquare and Fisher's exact tests. The continuous variables were tested for normality using the Kolmogorov-Smirnov Test. For the statistical correlation between two independent groups Mann-Whitney $U$ test was used for variables that do not show normal distribution, and Student's $T$ test was used for variables that show normal distribution. Statistically, P-values $<0.05$ were considered significant.

\section{RESULTS}

The mean age of the patients in the study group was 59.29 \pm 11.72 years (20-89 years), and the mean age of healthy individuals in the control group was $57.47 \pm 14.99$ years (2194 years). Of the 229 patients included in the study, 140 were male (61.1\%) and 89 (38.9\%) were female. Patient and control groups were comparable for age and gender ( $p>0.05)$.

Patients under two standard deviations of the mean total PMA in the control group were termed as sarcopenic. Accordingly, the determined cut-off values and sarcopenic patient numbers are presented in Table 1.
Demographic, clinical features and distribution of laboratory findings according to the presence of sarcopenia in patients with cirrhosis are presented in Table 2.

Although there was a statistically significant difference between sarcopenic and non-sarcopenic cirrhotic patients in terms of Child-Pugh prognostic scoring ( $p<0.001$ ); there was no significant difference for MELD scoring ( $p>0.05)$.

There was a statistically significant difference between the sarcopenic and non-sarcopenic cirrhotic patients in terms of 1-year and 3-year mortality ( $p<0.001$ and $p<0.01$, respectively). The rates of mortality at 1 and 3-year followups were significantly higher in sarcopenic cirrhotic patients (28.6\%, 40.7\%, respectively) than among non-sarcopenic cirrhotic patients ( $9.4 \%$ vs. $24.6 \%$, respectively).

\section{DISCUSSION}

Liver cirrhosis is an important cause of mortality in our country and diverse regions of the world. The most prevalent complications in cirrhosis are ascites, hepatic encephalopathy, varicose bleeding, susceptibility to infections, kidney dysfunction and HCC [3]. In addition, sarcopenia is another frequent complication of cirrhosis, which has a negative effect on survival, life quality and response to stress, such as infections and surgery [10]. Even though sarcopenia is mostly associated with aging, it can also result from chronic diseases, malignancy and 
Erkan et al. / Prognostic Value of Sarcopenia in Patients with Cirrhosis

Table 2. Distribution of Some Descriptive, Laboratory and Clinical Features According to the Presence of Sarcopenia in Patients with Cirrhosis

\begin{tabular}{|c|c|c|c|c|}
\hline & All patients $(n=229)$ & No sarcopenia $(n=138)$ & Sarcopenia $(n=91)$ & $\mathbf{p}$ \\
\hline Age (years) & $59.29 \pm 11.72(20-89)$ & $57.38 \pm 10.88(23-83)$ & $62.20 \pm 12.40(20-89)$ & $0.002^{*}$ \\
\hline \multicolumn{5}{|l|}{ Gender } \\
\hline Male & $140(61.1)$ & $89(64.5)$ & $51(56.0)$ & $0.199^{* *}$ \\
\hline Female & $89(38.9)$ & $49(35.5)$ & $40(44.0)$ & \\
\hline BMI $\left(\mathrm{kg} / \mathrm{m}^{2}\right)$ & $26.66 \pm 4.77(17.30-44.08)$ & $27.21 \pm 4.70(18.37-44.08)$ & $25.85 \pm 4.80(17.30-38.57)$ & $0.065^{* * *}$ \\
\hline \multicolumn{5}{|l|}{ Etiology of cirrhosis } \\
\hline HBV & $86(37.6)$ & $54(39.1)$ & $32(35.2)$ & \multirow{11}{*}{$0.564^{* *}$} \\
\hline $\mathrm{HCV}$ & $68(29.7)$ & $38(27.5)$ & $30(33.0)$ & \\
\hline Cryptogenic & $51(22.3)$ & $30(21.7)$ & $21(23.1)$ & \\
\hline Alcoholic & $11(4.8)$ & $9(6.5)$ & $2(2.2)$ & \\
\hline PBS & $4(1.7)$ & $3(2.2)$ & $1(1.1)$ & \\
\hline Wilson & $3(1.3)$ & $2(1.4)$ & $1(1.1)$ & \\
\hline Autoimmune hepatitis & $2(0.9)$ & $1(0.7)$ & $1(1.1)$ & \\
\hline Budd-Chiari & $1(0.4)$ & 0 & $1(1.1)$ & \\
\hline HDV & $1(0.4)$ & $1(0.7)$ & 0 & \\
\hline TBC & $1(0.4)$ & 0 & $1(1.1)$ & \\
\hline Congenital hepatic fibrosis & $1(0.4)$ & 0 & $1(1.1)$ & \\
\hline HCC & $48(21.0)$ & $25(18.1)$ & $23(25.3)$ & $0.193^{* *}$ \\
\hline Albumin & $3.52 \pm 0.65(1.60-5.20)$ & $3.63 \pm 0.59(1.60-4.61)$ & $3.34 \pm 0.68(1.70-5.20)$ & $<0.001^{* * *}$ \\
\hline Total Bilirubin & $1.80 \pm 2.34(0.3-21.6)$ & $1.69 \pm 2.02(0.3-15.9)$ & $1.97 \pm 2.75(0.3-21.6)$ & $0.603^{* * *}$ \\
\hline PT & $15.54 \pm 2.92(8.1-34.3)$ & $15.43 \pm 2.97(8.1-34.3)$ & $15.71 \pm 2.84(12.1-28.7)$ & $0.355^{* * *}$ \\
\hline INR & $1.33 \pm 0.33(0.62-3.44)$ & $1.32 \pm 0.33(0.62-3.44)$ & $1.35 \pm 0.33(0.99-3.15)$ & $0.333^{* * *}$ \\
\hline Creatinine & $0.77 \pm 0.46(0.26-6.21)$ & $0.73 \pm 0.22(0.27-1.40)$ & $0.82 \pm 0.68(0.26-6.21)$ & $0.830^{* * *}$ \\
\hline ALT & $53.76 \pm 54.41(6-625)$ & $57.03 \pm 63.02(10-625)$ & $48.80 \pm 37.60(6-201)$ & $0.179^{* * *}$ \\
\hline AST & $66.77 \pm 58.29(17-573)$ & $66.52 \pm 59.77(17-573)$ & $67.15 \pm 56.29(19-380)$ & $0.759^{* * *}$ \\
\hline Sodium & $137.1 \pm 3.9(111-144)$ & $137.7 \pm 2.5(130-144)$ & $136.1 \pm 5.2(111-144)$ & $0.028^{* * *}$ \\
\hline \multicolumn{5}{|l|}{ Ascites } \\
\hline None & $173(75.5)$ & $119(86.2)$ & $54(59.3)$ & \multirow{3}{*}{$<0.001^{* *}$} \\
\hline Mild & $37(16.2)$ & $14(10.1)$ & $23(25.3)$ & \\
\hline Moderate to severe & $19(8.3)$ & $5(3.7)$ & $14(15.4)$ & \\
\hline \multicolumn{5}{|l|}{\begin{tabular}{|l} 
Encephalopathy \\
\end{tabular}} \\
\hline None & $219(95.7)$ & $135(97.8)$ & $84(92.3)$ & \multirow{3}{*}{$0.110^{* *}$} \\
\hline Grade 1-2 & $9(3.9)$ & $3(2.2)$ & $6(6.6)$ & \\
\hline Grade 3-4 & $1(0.4)$ & 0 & $1(1.1)$ & \\
\hline
\end{tabular}

malnutrition, resulting in reduced functional capacity and high mortality risk in different patient groups $[1,11]$.

Malnutrition is among one of the most important causes of sarcopenia in patients with cirrhosis [12]. The prevalence of malnutrition in cirrhosis is $65 \%-90 \%$, and there are many reasons that cause malnutrition, of which low oral intake, malabsorption, metabolic abnormalities, increased energy requirement, accelerated protein degradation, insufficient protein synthesis and hypermetabolism are some of them [12]. However, evaluation of malnutrition status in cirrhotic patients is difficult due to the impact of hepatic synthetic dysfunction and salt lfluid accumulation in the body. Various methods have been developed to evaluate malnutrition in patients with cirrhosis; however, the shortage in objectivity, reproducibility and prognostic success restrict extensive implementation of those methods. Today, evaluating muscle mass with cross-sectional images acquired by $\mathrm{CT}$ or magnetic resonance imaging (MRI) is an objective and reproducible method for evaluating malnutrition and detecting sarcopenia [13]. It has been shown that the muscle area obtained from one abdominal section image acquired by CT or MRI has a strong correlation with the total body skeletal muscle mass measured on whole-body multi-slice imaging [14]. Cross-sectional muscle area calculated at the L3 level linearly associates with the whole-body muscle mass in the presence or absence of malignity, so the L3 vertebra level has often been used to evaluate muscle mass 
during the assessment of sarcopenia [14-20]. However, some studies have also measured the cross-sectional muscle area or psoas muscle area at the L4, L3-4 or umbilicus level [10,21-24]. Yoshizumi et al. demonstrated that body surface area, height, body weight and psoas muscle area at the L3 level correlate with the total muscle area [25]. Measuring the psoas muscle area on CT scan is simple and applicable in daily practice. Since muscle evaluation based on the crosssectional muscle area is complex and requires special software, psoas muscle assessments were initiated and found to be related with waiting list mortality and posttransplant mortality $[24,26]$. Therefore, in the present study, the psoas muscle area was measured at mid-L3 level on CT. However, due to differences in race and/or modalities, appropriate diagnostic cut-off values have not been established for existing methods. Unlike previous studies, we used a control group with similar race, age and gender distribution in this study to determine sarcopenic patients.

In our study, sarcopenia was found in $39.7 \%$ of cirrhotic patients. The prevalence of sarcopenia was found to be between $22.2 \%$ and $70 \%$ in 17 studies $[23,27]$. Similar to our results, Montano-Loza et al. reported that approximately $40 \%$ of cirrhotic patients had sarcopenia [16].

Prognostic evaluation is difficult in cirrhotic patients because the prognosis of these patients highly varies due to various factors, such as liver function capacity, presence and degree of portal hypertension, underlying cause of cirrhosis, reversibility of the underlying damage process and liver cancer formation [28]. Conventional prognostic scoring methods used for cirrhotic patients, such as CTP or MELD score, have limitations of including subjective data and not including extrahepatic prognostic data. In addition to these, the absence of malnutrition evaluation is an important deficit of those scoring methods.

In the current study, a statistically significant difference was found between patients with cirrhosis and those with and without sarcopenia in terms of Child-Pugh prognostic scoring; however, no significant difference was found in terms of MELD scoring. In some studies, while CTP or MELD scores correlate with sarcopenia, there are publications reporting that there is no correlation between sarcopenia and the CTP or MELD score [15-19]. The reason for that nonsignificant difference between sarcopenia and the MELD score in our study may be the lower average MELD scores.

Evaluation of sarcopenia in cirrhotic patients is a new and important predictor of survival. Montano-Loza et al. demonstrated that sarcopenia is associated with mortality in cirrhotic patients [16]. Transplant waiting list mortality was higher in patients with sarcopenia compared to those without sarcopenia in the study of Tandon et al. [17]. Additionally, in the study of Meza-Junco et al. sarcopenia was an independent predictor of mortality in patients evaluated for liver transplantation [18]. In consistent to the literature, in our study, among patients with cirrhosis with sarcopenia, the percentage of those who died at the end of 1 and 3 years of follow-up was significantly higher than that of patients without sarcopenia.

There are some limitations to be mentioned; the most important limitation of our study was that the patient population was obtained from a single centre and the number of patients was small. Another limitation of our study was that muscle strength or physical performance could not be evaluated due to the retrospective design of the study.

In conclusion, sarcopenia is one of the most common complications of cirrhosis and it is associated with increased mortality. While liver transplantation is the only treatment for cirrhosis, this is not possible in most of the patients. That's why, the management of cirrhotic patients is often focused on the prevention and control of complications. The results of this study suggest that psoas muscle atrophy shown by CT scan image at the level of the mid-L3 vertebra has predictive value for mortality in cirrhotic patients. Early identification of sarcopenic patients and starting the treatment early is vital for long life expectancy, improvement in quality of life and reduction of complications. Further studies including sarcopenia in conventional scores seems to provide better determination of mortality in cirrhotic patients.

Author contributions: All authors were involved in concept, design, collection of data, interpretation, writing, and critically revising the article. All authors approve final version of the article.

Funding: The authors received no financial support for the research and/or authorship of this article.

Declaration of interest: Authors declare no competing interest.

Data availability: Data generated or analysed during this study are available from the authors on request.

\section{REFERENCES}

1. Cruz-Jentoft AJ, Baeyens JP, Bauer JM, et al. European Working group on sarcopenia in older people. Sarcopenia: European consensus on definition and diagnosis: Report of the European Working Group on Sarcopenia in Older People. Age Ageing 2010; 39(4): 41223. (doi: 10.1093/ageing/afq034). 
2. Baumgartner RN, Koehler KM, Gallagher D, et al. Epidemiology of sarcopenia among the elderly in New Mexico. Am J Epidemiol 1998; 147: 755-63. (doi: 10.1093/oxfordjournals.aje.a009520).

3. Tsochatzis EA, Bosch J, Burroughs AK. Liver cirrhosis. Lancet 2014; 383: 1749-61. (doi: 10.1016/S01406736(14)60121-5)

4. Dasarathy S. Consilience in sarcopenia of cirrhosis. J Cachexia Sarcopenia Muscle 2012; 3: 225-37. (doi: 10.1007/s13539-012-0069-3).

5. Johnson TM, Overgard EB, Cohen AE, DiBaise JK. Nutrition assessment and management in advanced liver disease. Nutr Clin Pract 2013; 28: 15-29. (doi: 10.1177/0884533612469027).

6. O'Brien A, Williams R. Nutrition in end-stage liver disease: principles and practice. Gastroenterology 2008; 134: 1729-40. (doi: 10.1053/j.gastro.2008.02.001).

7. Thuluvath PJ, Triger DR. How valid are our reference standards of nutrition? Nutrition 1995; 11: 731-33.

8. Heymsfield SB, Casper K. Anthropometric assessment of the adult hospitalized patient. JPEN J Parenter Enteral Nutr 1987; 11(suppl): 36S-41S. (doi: 10.1177/014860718701100503).

9. Harries AD, Jones LA, Heatley RV, Rhodes J. Malnutrition in inflammatory bowel disease: an anthropometric study. Hum Nutr Clin Nutr 1982; 36: 307-13.

10. Englesbe MJ, Patel SP, He K, et al. Sarcopenia and mortality after liver transplantation. J Am Coll Surg 2010; 211: 271-78. (doi: 10.1016/j.jamcollsurg.2010.03.039).

11. Rosenberg $\mathrm{IH}$. Sarcopenia: origins and clinical relevance. J Nutr. 1997; 127(5 Suppl): 990S-1S.

12. Manguso F, D'ambra G, Menchise A, Sollazzo $R$, D'agostino L. Effects of an appropriate oral diet on the nutritional status of patients with Hcv-related liver cirrhosis: A prospective study. Clinical Nutrition 2005; 24: 751-59. (doi: 10.1016/j.clnu.2005.02.010).

13. Johnson TM, Overgard EB, Cohen AE, DiBaise JK. Nutrition assessment and management in advanced liver disease. Nutr Clin Pract 2013; 28: 15-29. (doi: 10.1177/0884533612469027).
14. Shen W, Punyanitya M, Wang Z, et al. Total body skeletal muscle and adipose tissue volumes: estimation from a single abdominal cross-sectional image. J Appl Physiol 2004; 97: 2333-38. (doi: 10.1152/japplphysiol.00744.2004).

15. Hanai T, Shiraki M, Nishimura K, et al. Sarcopenia impairs prognosis of patients with liver cirrhosis. Nutrition 2015; 31: 193-99. (doi: 10.1016/j.nut.2014.07.005).

16. Montano-Loza AJ, Meza-Junco J, Prado CM, et al. Muscle wasting is associated with mortality in patients with cirrhosis. Clin Gastroenterol Hepatol 2012; 10: 166-73. (doi: 10.1016/j.cgh.2011.08.028).

17. Tandon $P$, Ney M, Irwin I, et al. Severe muscle depletion in patients on the liver transplant wait list: its prevalence and independent prognostic value. Liver Transpl 2012; 18: 1209-216. (doi: 10.1002/lt.23495).

18. Meza-Junco J, Montano-Loza AJ, Baracos VE, et al. Sarcopenia as a prognostic index of nutritional status in concurrent cirrhosis and hepatocellular carcinoma. J Clin Gastroenterol 2013; 47: 861-70. (doi: 10.1097/MCG.0b013e318293a825).

19. Montano-Loza AJ, Meza-Junco J, Baracos VE, et al. Severe muscle depletion predicts postoperative length of stay but is not associated with survival after liver transplantation. Liver Transpl 2014; 20: 640-48. (doi: 10.1002/lt.23863).

20. Masuda T, Shirabe K, Ikegami T, et al. Sarcopenia is a prognostic factor in living donor liver transplantation. Liver Transpl 2014; 20: 401-07. (doi: 10.1002/lt.23811).

21. Krell RW, Kaul DR, Martin AR, et al. Association between sarcopenia and the risk of serious infection among adults undergoing liver transplantation. Liver Transpl 2013; 19: 1396-402. (doi: 10.1002/lt.23752).

22. DiMartini A, Cruz RJ, Dew MA, et al. Muscle mass predicts outcomes following liver transplantation. Liver Transpl 2013; 19: 1172-180. (doi: 10.1002/lt.23724).

23. Cruz RJ, Dew MA, Myaskovsky L, et al. Objective radiologic assessment of body composition in patients with end-stage liver disease: going beyond the BMI. Transplantation 2013; 95: 617-22. (doi: 10.1097/TP.0b013e31827a0f27). 
24. Durand F, Buyse S, Francoz C, et al. Prognostic value of muscle atrophy in cirrhosis using psoas muscle thickness on computed tomography. J Hepatol 2014; 60: 1151-157. (doi: 10.1016/j.jhep.2014.02.026).

25. Yoshizumi T, Shirabe K, Nakagawara H, et al. Skeletal muscle area correlates with body surface area in healthy adults. Hepatol Res. 2014; 44: 313-18. (doi: 10.1111/hepr.12119).

26. Kim TY, Kim MY, Sohn JH, et al. Sarcopenia as a useful predictor for long-term mortality in cirrhotic patients with ascites. J Korean Med Sci 2014; 29: 1253-259. (doi: 10.3346/jkms.2014.29.9.1253).
27. Yadav A, Chang YH, Carpenter S, et al. Relationship between sarcopenia, six-minute walk distance and health-related quality of life in liver transplant candidates. Clin Transplant 2015; 29: 134-41. (doi: 10.1111/ctr.12493).

28. Durand F, Valla D. Assessment of prognosis of cirrhosis. Semin Liver Dis 2008; 28: 110-22. (10.1055/s-20081040325). 